\title{
Utilización del simulador EPANET en el análisis de redes de agua potable en edificaciones
}

\author{
Aguirre F.*; Ordoñez J.**; Oyola J.**; Romero E.** \\ Universidad Técnica de Machala, Facultad de Ingeniería Civil, Machala, Ecuador \\ e-mail: faguirre@utmachala.edu.ec
}

\section{RESUMEN}

EPANET es un modelo de simulación por computador muy conocido para el estudio de redes de distribución de agua potable en sistemas públicos. No existe suficiente información de su utilización en redes de distribución de agua en edificaciones, por lo que resulta interesante simular el comportamiento hidráulico de este tipo de redes de suministro a presión.

El programa original no está concebido para analizar redes de distribución en edificios, porque los caudales demandados por los aparatos sanitarios se basan en la probabilidad de su uso simultaneo, es decir es poco probable que todos funcionen al mismo tiempo. En edificaciones no se cumple la ecuación de continuidad en cada nudo como en las redes de distribución públicas.

El presente artículo presenta una metodología subsanando el desbalance de caudales en los nudos de la red, dotando al proyectista de una herramienta con un software libre que permite de forma rápida calcular la red, realizando simulaciones en múltiples escenarios en busca de una optimización hidráulica hasta cumplir con lo establecido en la Norma Hidrosanitaria del Ecuador.

Esta metodología fue utilizada en el estudio de una red de distribución de agua potable en un edificio de 3 pisos abastecida desde un tanque elevado, con 15 aparatos sanitarios, se consideraron las pérdidas de energía por fricción y locales, para analizar las velocidades del agua en las tuberías, las presiones de servicio en cada aparato sanitario y simular su comportamiento hidráulico.

En base a los resultados obtenidos se observa que se cumplen con los requerimientos establecidos en la normativa que establecen velocidades mínimas de $0.60 \mathrm{~m} / \mathrm{s}$ y que las presiones sean mayores a $3 \mathrm{~m}$. en todos los aparatos sanitarios.

Palabras clave: EPANET, modelación hidráulica, redes de agua potable interiores. 


\section{ABSTRACT}

EPANET is a well-known computer simulation model for the study of drinking wáter distribution networks in public systems. There is not enough information on its use in water distribution networks in buildings, so it is interesting to simulate the hydraulic behavior of this type of pressure supply networks.

The original program is not designed to analyze distribution networks in buildings, because the flows demanded by the sanitary devices are based on the probability of their simultaneous use, that is, it is unlikely that all will work at the same time. In buildings the continuity equation is not fulfilled in each node as in the public distribution networks.

This article presents a methodology correcting the imbalance of flows in the nodes of the network, providing the designer with a tool with free software that allows you to quickly calculate the network, performing simulations in multiple scenarios in search of a hydraulic optimization to meet with what is established in the Hydrosanitary Standard of Ecuador.

This methodology was used in the study of a drinking water distribution network in a three apartment building supplied from an elevated tank, with 15 sanitary appliances, friction and local energy losses were considered, to analyze the water velocities in the pipes, the pressures in each sanitary appliance and simulate its hydraulic behavior.

Based on the results obtained, it is observed that the requirements established in the regulations that establish minimum speeds of $0.60 \mathrm{~m} / \mathrm{s}$ are met and that the pressures are greater than $3 \mathrm{~m}$. in all sanitary appliances.

\section{Keywords:}

EPANET, hydraulic modeling, indoor pipe network analysis

\section{Introducción}

El objetivo del presente trabajo es diseñar y modelar la red de distribución de agua potable de una edificación multifamiliar de tres pisos abastecido desde un tanque elevado, usando en software libre EPANET, comprobando que los resultados obtenidos cumplan con los requerimientos mínimos establecidos en la Norma Hidrosanitaria NHE del Ecuador.

Un sistema de distribución de agua es una estructura hidráulica que contienen elementos tales como tuberías, tanques, bombas, válvulas, etc. Es muy importante suministrar agua potable a los usuarios en la cantidad suficiente y la presión adecuada, por lo que es un desafío para los diseñadores obtener soluciones técnicas adecuadas para cada proyecto, cumpliendo con las normas requeridas. (Venkata, Sudheer, \& Rajasekhar, 2015) 
En base a la información de las características de la tubería, bombas, tanques elevados y del consumo de agua en los domicilios, el análisis hidráulico por computadora puede simplificar esta tarea. El uso de programas para el diseño y modelado aumenta cada día, al utilizar programas de dominio público como EPANET, Branch, LOOP, HidrasSoftware, NETIS, Giswater, tanto como los programas comerciales como WaterCAD, WaterGems y otros. (Morelos \& Ramírez, 2017)

La red de distribución corresponde a un edificio proyectado en la ciudad de Machala, provincia de El Oro, en la que la presión en la red de distribución no tiene la presión suficiente para abastecer directamente a los edificios por lo que se hace necesario diseñar un sistema indirecto con cisterna, equipo de bombeo, tanque de reserva elevado y la red de distribución.

EPANET es un programa orientado al análisis del comportamiento de los sistemas de distribución de agua y el seguimiento de la calidad del agua en los mismos, que ha tenido una gran aceptación en todo el mundo, por ser gratis y muy sencillo de utilizar. (Shivalingaswami, Vijaykumar, \& Nagaraj, 2016)

EI EPANET es un programa que realiza modelaciones de redes de agua potable, que analiza el comportamiento hidráulico y la calidad del agua en las redes de tuberías que trabajan a presión. Una red está conformada por tuberías, nudos (uniones), bombas, válvulas y depósitos de almacenamiento o embalses. A medida que el tiempo ha pasado se han desarrollado metodologías para la modelación de la demanda de agua aplicando métodos estocásticos, definiendo tres características dinámicas para el consumo de agua como: intensidad, duración y frecuencia de uso. (Saldarriaga, Modelo estocastico de demanda de agua en edificaciones y la recoleccion y procesamieno de datos para su formulacion, 2014)

El uso de agua y sus distintas aplicaciones en el medio han convertido el estudio de distribución de esta en la pieza principal para la elaboración de distintos métodos que satisfagan las necesidades de consumo de agua. "El agua potable es un elemento necesario e indispensable para las actividades diarias del ser humano" (Miranda Trejo, Ocampo Fletes, Escobedo Castillo, \& Hernandez Rodriguez, 2015)

Los modelos existentes en el medio han sido elaborados en países desarrollados donde el servicio del agua potable es continuo, por ende, la demanda del agua es continua y no se crea una inestabilidad de consumo, solo basta con realizar la acometida del sistema para obtener el líquido vital" (Cabrera Bejar \& Gueorguiev Tzatchkov, 2012)

La función principal de las redes de distribución de agua es la de suministrar agua en la cantidad suficiente para abastecer la demanda y con la calidad requerida en las normas nacionales, por lo que representa un desafío para los técnicos encargados de diseñar este tipo de obras. (Mehta, Lakhani, Patel, \& Patel, 2015) 
La red de distribución analizada esta abastecida desde un tanque elevado, una columna descendente, tres ramales y quince aparatos sanitarios, se dimensionará la red tal que, bajo condiciones normales de funcionamiento, provea los caudales instantáneos y presiones mínimas según la norma establecida.

Los caudales de cada aparato y las presiones mínimas se muestran en la Tabla 1.

Tabla 1 Caudales y presiones en aparatos de consumo (NEC, 2011)

\begin{tabular}{|l|c|c|c|}
\hline \multirow{2}{*}{ Aparato sanitario } & \multirow{2}{*}{$\begin{array}{c}\text { Caudal } \\
\text { instantáneo } \\
\text { mínimo }(\mathrm{I} / \mathrm{s})\end{array}$} & $\begin{array}{c}\text { Pecomendada } \\
\text { (m.c.a.) }\end{array}$ & Mínima (m.c.a.) \\
\cline { 3 - 4 } & 0.10 & 5.00 & 2.00 \\
\hline Lavamanos & 0.10 & 7.00 & 3.00 \\
\hline Inodoro con deposito & 0.20 & 10.00 & 3.00 \\
\hline Ducha & 0.20 & 5.00 & 2.00 \\
\hline Fregadero cocina & 0.20 & 7.00 & 3.00 \\
\hline Lavadora & \multicolumn{2}{|}{} \\
\hline
\end{tabular}

El caudal de cálculo de cada tramo de red es igual al caudal máximo probable que se determina con la siguiente ecuación:

$$
Q_{M P}=k_{s} \cdot \sum Q_{i}
$$

$\boldsymbol{Q}_{\boldsymbol{M P}}=$ caudal máximo probable $(\mathrm{l} / \mathrm{s})$

$\boldsymbol{Q}_{i}=$ caudal mínimo de los aparatos suministrados (l/s)

$\boldsymbol{k}_{\boldsymbol{s}}=$ Coeficiente de simultaneidad, entre 0.2 y 1.0

$$
k_{s}=\frac{1}{\sqrt{n-1}}+F(0.04+0.04 . \log (\log (n)))
$$

$\boldsymbol{n}=$ número de aparatos servidos

$\boldsymbol{F}=2$, para edificios habitacionales

Para el caso de las redes de distribución de agua potable en sistemas públicos la sumatoria de caudales que ingresan a un nudo es igual a los caudales que salen de él, en el caso de las redes en edificaciones la ecuación de continuidad en cada nudo no se cumple debido a al coeficiente de simultaneidad $\boldsymbol{k}_{\boldsymbol{s}}$ que depende del número de aparatos servidos. (Blokker, Vreeburg, Buchberger, \& van Dijk, 2008)

En vista de esta inconveniente se ha desarrollado una metodología que permita utilizar el programa EPANET, para analizar y modelar redes de distribución de agua potable en edificaciones abastecidas desde tanques elevados. 


\section{Materiales y Métodos}

Se elaboro la isometría de la red en base a la información de los planos arquitectónicos, determinando las longitudes de cada uno de los tramos de tubería, se asignaron los caudales de los aparatos sanitarios de acuerdo con la Tabla 2. Además, se identificaron todos los nudos de la red.

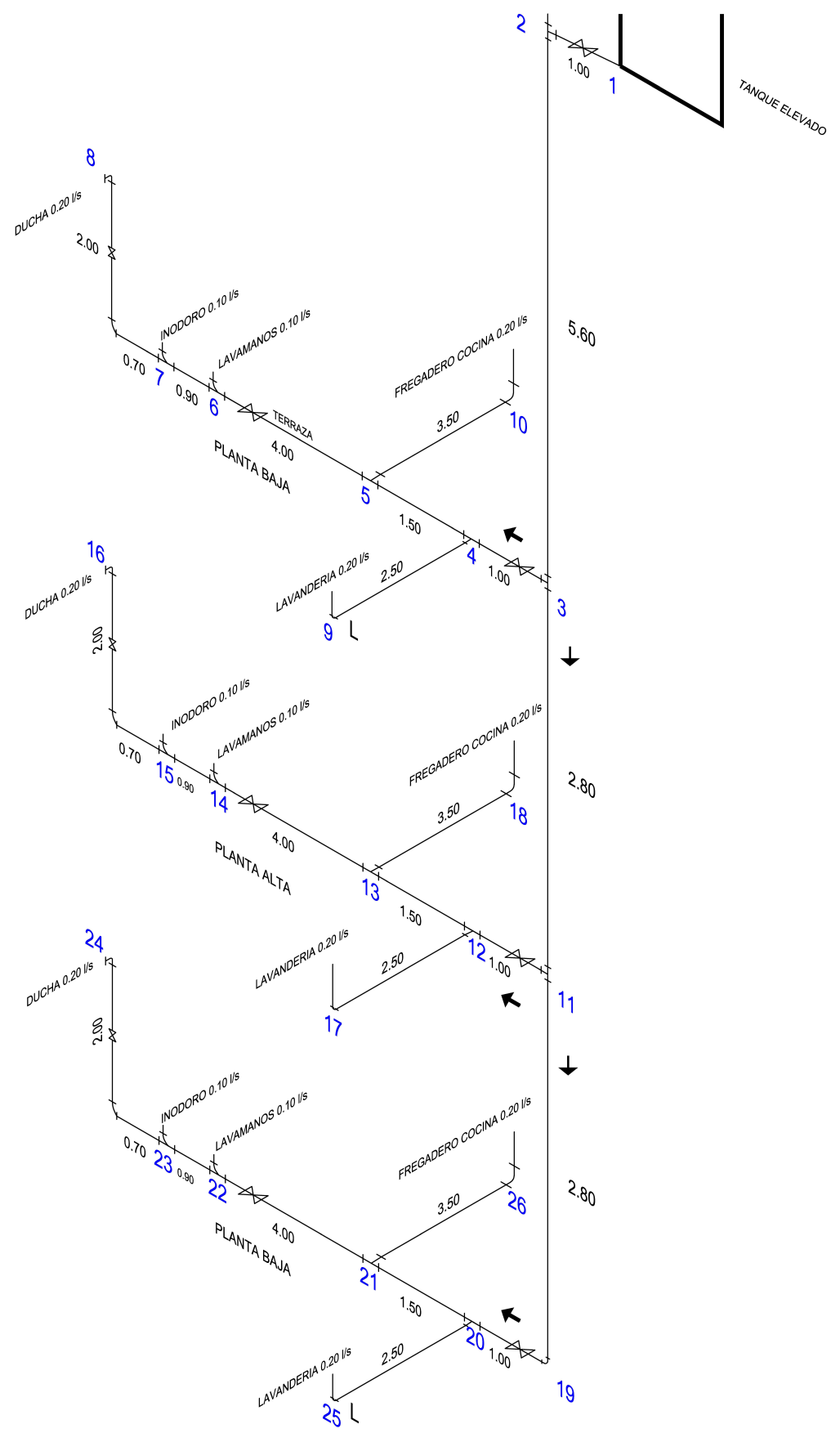

Figura 1 Isometría de la red de distribución 
Una de las diferencias fundamentales entre una red de distribución de agua potable en las ciudades con otra red en edificios es que la primera se cumple que el caudal que ingresa a un nudo es igual a los caudales que salen del mismo nudo, en cambio en la segunda este balance no se cumple debido a la simultaneidad en el uso de los aparatos sanitarios. (Adeniran \& Oyelowo, 2014)

Para cada tramo el caudal máximo posible $\sum \boldsymbol{Q}_{i}$ fue calculado, posteriormente se calculó el coeficiente de simultaneidad y finalmente el caudal máximo probable. Los resultados obtenidos con el software Excel se muestran en la Tabla 3.AW E6

Tabla 4 Caudal máximo probable en la red

\begin{tabular}{|c|c|c|c|c|c|}
\hline Nudo inicial & Nudo final & $\begin{array}{c}\text { Numero de } \\
\text { aparatos sanitarios } \\
\text { servidos por el } \\
\text { tramo }\end{array}$ & $\begin{array}{l}\text { Caudal máximo } \\
\text { posible } \sum \text { Qi (I/s) }\end{array}$ & $\begin{array}{c}\text { Factor de } \\
\text { Simultaneidad } \\
\text { ks }\end{array}$ & $\begin{array}{c}\text { Caudal } \\
\text { máximo } \\
\text { probable } \\
\mathbf{Q}_{\text {MP }}(\mathrm{l} / \mathrm{s})\end{array}$ \\
\hline 1 & 2 & 15 & 0.00 & 0.27 & 0.00 \\
\hline 2 & 3 & 15 & 1.80 & 0.27 & 0.49 \\
\hline 3 & 4 & 5 & 0.20 & 0.50 & 0.10 \\
\hline 4 & 5 & 4 & 0.30 & 0.58 & 0.17 \\
\hline 5 & 6 & 3 & 0.10 & 0.71 & 0.07 \\
\hline 6 & 7 & 2 & 0.10 & 1.00 & 0.10 \\
\hline 7 & 8 & 1 & 0.20 & 1.00 & 0.20 \\
\hline 4 & 9 & 1 & 0.20 & 1.00 & 0.20 \\
\hline 5 & 10 & 1 & 0.20 & 1.00 & 0.20 \\
\hline 3 & 11 & 10 & 1.80 & 0.34 & 0.61 \\
\hline 11 & 12 & 5 & 0.90 & 0.50 & 0.45 \\
\hline 12 & 13 & 4 & 0.70 & 0.58 & 0.41 \\
\hline 13 & 14 & 3 & 0.40 & 0.71 & 0.28 \\
\hline 14 & 15 & 2 & 0.30 & 1.00 & 0.30 \\
\hline 15 & 16 & 1 & 0.20 & 1.00 & 0.20 \\
\hline 12 & 17 & 1 & 0.20 & 1.00 & 0.20 \\
\hline 13 & 18 & 1 & 0.20 & 1.00 & 0.20 \\
\hline 11 & 19 & 5 & 0.90 & 0.50 & 0.45 \\
\hline 19 & 20 & 5 & 0.90 & 0.50 & 0.45 \\
\hline 20 & 21 & 4 & 0.70 & 0.58 & 0.41 \\
\hline 21 & 22 & 3 & 0.40 & 0.71 & 0.28 \\
\hline 22 & 23 & 2 & 0.30 & 1.00 & 0.30 \\
\hline 23 & 24 & 1 & 0.20 & 1.00 & 0.20 \\
\hline 20 & 25 & 1 & 0.20 & 1.00 & 0.20 \\
\hline 21 & 26 & 1 & 0.20 & 1.00 & 0.20 \\
\hline
\end{tabular}

Conference Proceeding UTMACH 2020 Vol.4, $\mathrm{n}^{\circ} 1$ 
Esta simultaneidad dificulta el ingreso de caudales en el modelo EPANET, tomando como referencia el nudo 5, los caudales de salida son $0.30 \mathrm{l} / \mathrm{s}$ y $0.20 \mathrm{l} / \mathrm{s}$ respectivamente, para que se cumpla el principio de conservación de la masa el caudal de entrada debería ser $0.50 \mathrm{l} / \mathrm{s}$, pero si se considera la simultaneidad es $0.45 \mathrm{l} / \mathrm{s}$. (ver Figura 2).

Para obviar este inconveniente al momento del ingreso de los caudales en cada nudo de la red en el modelo, se debe ingresar un caudal ficticio que permita que el caudal que ingresa a cada nudo sea el máximo probable calculado para el tramo analizado. Para el nudo 5, el caudal ficticio es $-0.05 \mathrm{l} / \mathrm{s}$. Con lo que se cumple que el caudal de entrada $0.45 \mathrm{l} / \mathrm{s}$ es igual a la sumatoria de los de salida $(0.30+0.20-0.05)$ I/s. (Conety, Renganathan, Perumal, \& Paez, 2019)

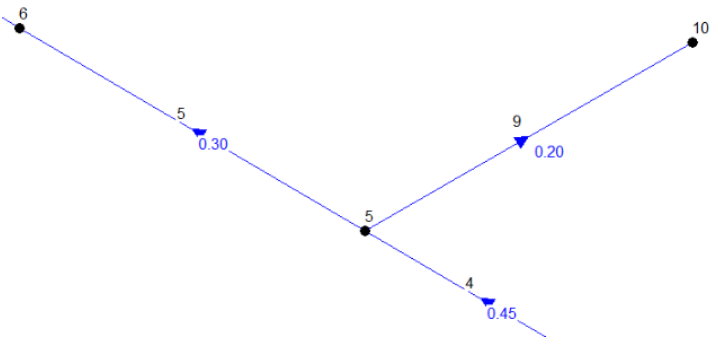

Figura 3 Caudales en el nudo 5

Con los valores del caudal máximo probable, el diámetro inicial de cada tramo fue determinado aplicando la ecuación de continuidad asumiendo una velocidad media de $1.0 \mathrm{~m} / \mathrm{s}$.

Para calcular la perdida de energía por fricción se utilizó le ecuación de Darcy - Weisbach, que teóricamente es la más recomendada y es aplicable a diámetros desde 0.0122 hasta $0.50 \mathrm{~m}$ como es el caso del proyecto estudiado.

$$
h_{f}=f \frac{L}{D} \frac{V^{2}}{2 g}
$$

Reemplazando $\boldsymbol{V}=\frac{4 \boldsymbol{Q}}{\pi D^{2}}$ en la ecuación 3 , se obtiene la ecuación 4 , que permite calcular directamente la perdida de carga.

$$
h_{f}=\frac{8 f L Q_{M P}^{2}}{\pi^{2} g D^{5}}
$$

Donde:

$\boldsymbol{h}_{\boldsymbol{f}}=$ pérdida de carga $(\mathrm{m})$.

$\boldsymbol{f}=$ factor de friccion (adimensional)

$\boldsymbol{L}=$ longitud de la tuberia, $(\mathrm{m})$.

Conference Proceeding UTMACH 2020 Vol. $4, \mathrm{n}^{\circ} 1$ 
$\boldsymbol{Q}=$ caudal, $\left(\mathrm{m}^{3} / \mathrm{s}\right)$.

$\boldsymbol{V}=$ velocidad de flujo, $(\mathrm{m} / \mathrm{s})$.

$\boldsymbol{g}=$ aceleracion de la gravedad, $\left(9.81 \mathrm{~m}^{2} / \mathrm{s}\right)$.

$\boldsymbol{D}=$ diametro de la tuberia, $(\mathrm{m})$.

El factor de fricción depende de la rugosidad absoluta de la tubería $\varepsilon$, del diámetro $D$ y del Numero de Reynolds, Re.

El Número de Reynolds, es un grupo adimensional, viene dado por el cociente de las fuerzas de inercia por las fuerzas debidas a la viscosidad.

Para tuberías circulares y flujo a tubería llena, el número de Reynolds se calcula de la siguiente manera:

Donde:

$$
R_{e}=\frac{V D}{v}=\frac{4 Q}{\pi D v}
$$

$\mathrm{V}=$ velocidad media $(\mathrm{m} / \mathrm{s})$

$\mathrm{D}=$ diámetro de la tubería $(\mathrm{m})$

$v=$ viscosidad cinemática del agua $\left(1.003 \times 10^{-6} \mathrm{~m}^{2} / \mathrm{s}\right.$ a $\left.20{ }^{\circ} \mathrm{C}\right)$

Para flujo turbulento, es decir cuando el número de Reynolds $\operatorname{Re}>4000$ se puede emplear ecuación explicita de Swamee - Jain con la que se obtiene resultados aproximados a los de la ecuación de Colebrook - White. (Venkata, Sudheer, \& Pradad, Hydraulic Simulation of Existing Water Distribution System using EPANET, 2016)

La fórmula de Swamee - Jain es la siguiente:

$$
f=\frac{0.25}{\left[\log _{10}\left(\frac{\varepsilon}{3.7 D}+\frac{5.74}{R e^{0.9}}\right)\right]^{2}}
$$

Donde:

$f=$ factor de fricción

$\varepsilon=$ rugosidad absoluta $(0.0015 \mathrm{~mm}$ para tuberías de PVC tipo roscable)

$\mathrm{D}=$ diámetro interno de la tubería $(\mathrm{m})$

$\mathrm{Re}=$ Número de Reynolds

Las pérdidas menores, también llamadas locales pueden interpretarse como debidas al incremento de la turbulencia que se produce en los cambios de dirección, codos, accesorios, etc.

El valor de la perdida menor o local es el producto del coeficiente de pérdidas menores por la altura de velocidad de la tubería, esto es: (Aguirre, 2015) 


$$
\boldsymbol{h}_{m}=K \frac{V^{2}}{2 \boldsymbol{g}}=\frac{8 K Q_{M P}^{2}}{\pi^{2} g D^{4}}
$$

$K=$ coeficiente de pérdidas menores

$V=$ velocidad del flujo $(\mathrm{m} / \mathrm{s})$

$Q=$ caudal $\left(\mathrm{m}^{3} / \mathrm{s}\right)$

$\mathrm{g}=$ aceleración de la gravedad, $9.81 \mathrm{~m} / \mathrm{s}^{2}$

$D=$ diametro $(\mathrm{m})$

En la siguiente tabla, se proporciona el valor del coeficiente de perdidas menores para algunos de los accesorios más comunes.

Tabla 5 Coeficientes de perdidas menores en accesorios (Saldarriaga, Hidráulica de Tuberías, Abastecimiento de Agua, Redes, Riegos, 2015)

\begin{tabular}{|l|c|}
\hline \multicolumn{1}{|c|}{ Accesorio } & $\mathrm{K}$ \\
\hline Válvula de globo, completamente abierta & 10.0 \\
\hline Válvula de cheque, completamente abierta & 2.5 \\
\hline Válvula de compuerta, completamente abierta & 0.2 \\
\hline Codo de radio corto & 0.9 \\
\hline Codo de 45o & 0.4 \\
\hline Te, en sentido recto & 0.3 \\
\hline Te, a través de la salida lateral & 1.8 \\
\hline Unión & 0.3 \\
\hline Entrada recta a tope & 0.5 \\
\hline Entrada con boca acampanada & 0.1 \\
\hline Entrada con tubo entrante & 0.9 \\
\hline Salida & 1.0 \\
\hline
\end{tabular}

Los datos ingresados al modelo para los tramos de tuberías se muestran en la tabla

Tabla 6 Datos de las tuberías y los nudos

\begin{tabular}{|r|r|r|r|r|r|r|r|}
\hline \multicolumn{5}{|c|}{ DATOS DE LOS TRAMOS DE TUBERIA } & \multicolumn{3}{|c|}{ DATOS DE LOS NUDOS } \\
\hline Línea & $\begin{array}{c}\text { Nudo } \\
\text { Inicial }\end{array}$ & $\begin{array}{c}\text { Nudo } \\
\text { Final }\end{array}$ & $\begin{array}{c}\text { Longitud } \\
\mathrm{m}\end{array}$ & $\begin{array}{c}\text { Diámetro } \\
\mathrm{mm}\end{array}$ & Nudo & $\begin{array}{c}\text { Demanda } \\
\text { Ficticia (I/s) }\end{array}$ & $\begin{array}{c}\text { Demanda } \\
\text { Real) } \mathrm{l} / \mathrm{s}\end{array}$ \\
\hline 1 & 1 & 2 & 1 & 49.10 & 1 & -0.95 & \\
\hline 2 & 2 & 3 & 5.6 & 37.92 & 2 & 0.00 & \\
\hline 3 & 3 & 4 & 1 & 32.35 & 3 & -0.30 & \\
\hline
\end{tabular}

Conference Proceeding UTMACH 2020 Vol.4, nº1 


\begin{tabular}{|r|r|r|r|r|r|r|r|}
\hline 4 & 4 & 5 & 1.5 & 32.35 & 4 & -0.14 & \\
\hline 5 & 5 & 6 & 4 & 24.20 & 5 & -0.05 & 0.10 \\
\hline 6 & 6 & 7 & 0.9 & 24.20 & 6 & 0.00 & 0.10 \\
\hline 7 & 7 & 8 & 2.7 & 18.76 & 7 & 0.10 & 0.10 \\
\hline 8 & 4 & 9 & 1 & 18.76 & 8 & 0.20 & 0.20 \\
\hline 9 & 5 & 10 & 3.5 & 18.76 & 9 & 0.20 & 0.20 \\
\hline 10 & 3 & 11 & 2.8 & 24.20 & 10 & 0.20 & 0.20 \\
\hline 11 & 11 & 12 & 1 & 18.76 & 11 & -0.28 & \\
\hline 12 & 12 & 13 & 1.5 & 18.76 & 12 & -0.14 & \\
\hline 13 & 13 & 14 & 4 & 18.76 & 13 & -0.05 & 0.10 \\
\hline 14 & 14 & 15 & 0.9 & 18.76 & 14 & 0.00 & 0.10 \\
\hline 15 & 15 & 16 & 2.7 & 13.79 & 15 & 0.10 & 0.10 \\
\hline 16 & 12 & 17 & 2.5 & 18.76 & 16 & 0.20 & 0.20 \\
\hline 17 & 13 & 18 & 3.5 & 18.76 & 17 & 0.20 & 0.20 \\
\hline 18 & 11 & 19 & 2.8 & 18.76 & 18 & 0.20 & 0.20 \\
\hline 19 & 19 & 20 & 1 & 18.76 & 19 & 0.00 & \\
\hline 20 & 20 & 21 & 1.5 & 18.76 & 20 & -0.14 & \\
\hline 21 & 21 & 22 & 4 & 18.76 & 21 & -0.05 & 0.10 \\
\hline 22 & 22 & 23 & 0.9 & 13.79 & 22 & 0.00 & 0.10 \\
\hline 23 & 23 & 24 & 2.7 & 13.79 & 23 & 0.10 & 0.10 \\
\hline 24 & 20 & 25 & 2.5 & 18.76 & 24 & 0.20 & 0.20 \\
\hline 25 & 21 & 26 & 3.5 & 18.76 & 25 & 0.20 & 0.20 \\
\hline & & & & & 26 & 0.20 & 0.20 \\
\hline
\end{tabular}

Con los datos de la isometría de la red, cotas y caudales de cada nudo; longitud, diámetro interior y rugosidad de la tubería en cada tramo, se ejecutó la modelación hidráulica de la red de distribución del edificio, para obtener las pérdidas de carga, velocidades y presiones, que permitan observar su comportamiento y verificar el cumplimiento de los requisitos establecidos en la NEC.

\section{Discusión de Resultados}

Una vez cargados los datos en el programa se realizó la modelación hidráulica, obteniendo los siguientes resultados:

En la Tabla 7 se muestran los valores calculados de la altura piezométrica y la presión en cada nudo, observando que en el nudo critico que corresponde al número 8, por estar ubicado en el lugar más alto de la instalación la presión calculada es de $3.04 \mathrm{~m}$, mayor al mínimo establecido de $3.00 \mathrm{~m}$. En todos los nudos restantes a excepción de los nudos 1 y 2 , al estar ubicados en puntos más bajos del nudo 3 , la presión en mayor a la mínima recomendada. 
Para el caso del nudo 1 indudablemente la presión es igual a 0 , que corresponde a la presión en la superficie del agua en el tanque. El nudo 2 tiene una presión de 0.06 $\mathrm{m}$ por estar muy cerca a la salida del reservorio de agua.

Tabla 8 Resultados de los nudos

\begin{tabular}{|c|c|c|c|}
\hline Nudo & $\begin{array}{c}\text { Demanda } \\
(\mathrm{I} / \mathrm{s})\end{array}$ & $\begin{array}{c}\text { Altura } \\
\text { piezométrica } \\
(\mathrm{m})\end{array}$ & Presión (m) \\
\hline 1 & -0.95 & 11.30 & 0.00 \\
\hline 2 & 0.00 & 11.26 & 0.06 \\
\hline 3 & -0.30 & 11.08 & 5.48 \\
\hline 4 & -0.14 & 11.02 & 5.42 \\
\hline 5 & -0.05 & 10.98 & 5.38 \\
\hline 6 & 0.00 & 10.84 & 5.24 \\
\hline 7 & 0.10 & 10.78 & 5.18 \\
\hline 8 & 0.20 & 10.64 & 3.04 \\
\hline 9 & 0.20 & 10.96 & 5.36 \\
\hline 10 & 0.20 & 10.81 & 5.21 \\
\hline 11 & -0.28 & 10.50 & 7.70 \\
\hline 12 & -0.14 & 9.97 & 7.17 \\
\hline 13 & -0.05 & 9.47 & 6.67 \\
\hline 14 & 0.00 & 9.03 & 6.23 \\
\hline 15 & 0.10 & 8.85 & 6.05 \\
\hline 16 & 0.20 & 8.27 & 3.47 \\
\hline 17 & 0.20 & 9.85 & 7.05 \\
\hline 18 & 0.20 & 9.31 & 6.51 \\
\hline 19 & 0.00 & 9.75 & 9.75 \\
\hline 20 & -0.14 & 9.22 & 9.22 \\
\hline 21 & -0.05 & 8.72 & 8.72 \\
\hline 22 & 0.00 & 8.28 & 8.28 \\
\hline 23 & 0.10 & 7.59 & 7.59 \\
\hline 24 & 0.20 & 7.01 & 5.01 \\
\hline 25 & 0.20 & 9.10 & 9.10 \\
\hline 26 & 0.20 & 8.56 & 8.56 \\
\hline
\end{tabular}

Los resultados de los tramos se muestran en la Tabla 9, la velocidad media del agua en las tuberías es mayor a $0.60 \mathrm{~m} / \mathrm{s}$ que es la mínima recomendada, a excepción del tramo inicial que tiene un valor de $0.50 \mathrm{~m} / \mathrm{s}$.

Tabla 10 Resultados en los tramos

\begin{tabular}{|c|c|c|c|}
\hline Tramo & Caudal $(\mathrm{I} / \mathrm{s})$ & $\begin{array}{c}\text { Velocidad } \\
(\mathrm{m} / \mathrm{s}) .\end{array}$ & $\begin{array}{c}\text { Perdida de } \\
\text { carga } \\
\text { unitaria } \\
(\mathrm{m} / \mathrm{km})\end{array}$ \\
\hline
\end{tabular}

Conference Proceeding UTMACH 2020 Vol. $4, \mathrm{n}^{\circ} 1$ 


\begin{tabular}{|r|r|r|r|}
\hline 1 & 0.95 & 0.50 & 35.95 \\
\hline 2 & 0.95 & 0.84 & 33.72 \\
\hline 3 & 0.51 & 0.62 & 51.09 \\
\hline 4 & 0.45 & 0.55 & 31.01 \\
\hline 5 & 0.30 & 0.65 & 34.57 \\
\hline 6 & 0.30 & 0.65 & 68.15 \\
\hline 7 & 0.20 & 0.72 & 51.88 \\
\hline 8 & 0.20 & 0.72 & 65.02 \\
\hline 9 & 0.20 & 0.72 & 47.87 \\
\hline 10 & 0.74 & 1.61 & 206.33 \\
\hline 11 & 0.51 & 1.85 & 524.51 \\
\hline 12 & 0.45 & 1.63 & 332.16 \\
\hline 13 & 0.30 & 1.09 & 110.29 \\
\hline 14 & 0.30 & 1.09 & 203.30 \\
\hline 15 & 0.20 & 1.34 & 214.37 \\
\hline 16 & 0.20 & 0.72 & 50.62 \\
\hline 17 & 0.20 & 0.72 & 47.87 \\
\hline 18 & 0.51 & 1.85 & 268.12 \\
\hline 19 & 0.51 & 1.85 & 524.51 \\
\hline 20 & 0.45 & 1.63 & 332.16 \\
\hline 21 & 0.30 & 1.09 & 110.29 \\
\hline 22 & 0.30 & 2.01 & 772.75 \\
\hline 23 & 0.20 & 1.34 & 214.37 \\
\hline 24 & 0.20 & 0.72 & 50.62 \\
\hline 25 & 0.20 & 0.72 & 47.87 \\
\hline & & & \\
\hline
\end{tabular}

En la Figura 4, se observa que la presión en los nudos $3,4,5,6,7$ y 8 , es mayor a la mínima recomendada.

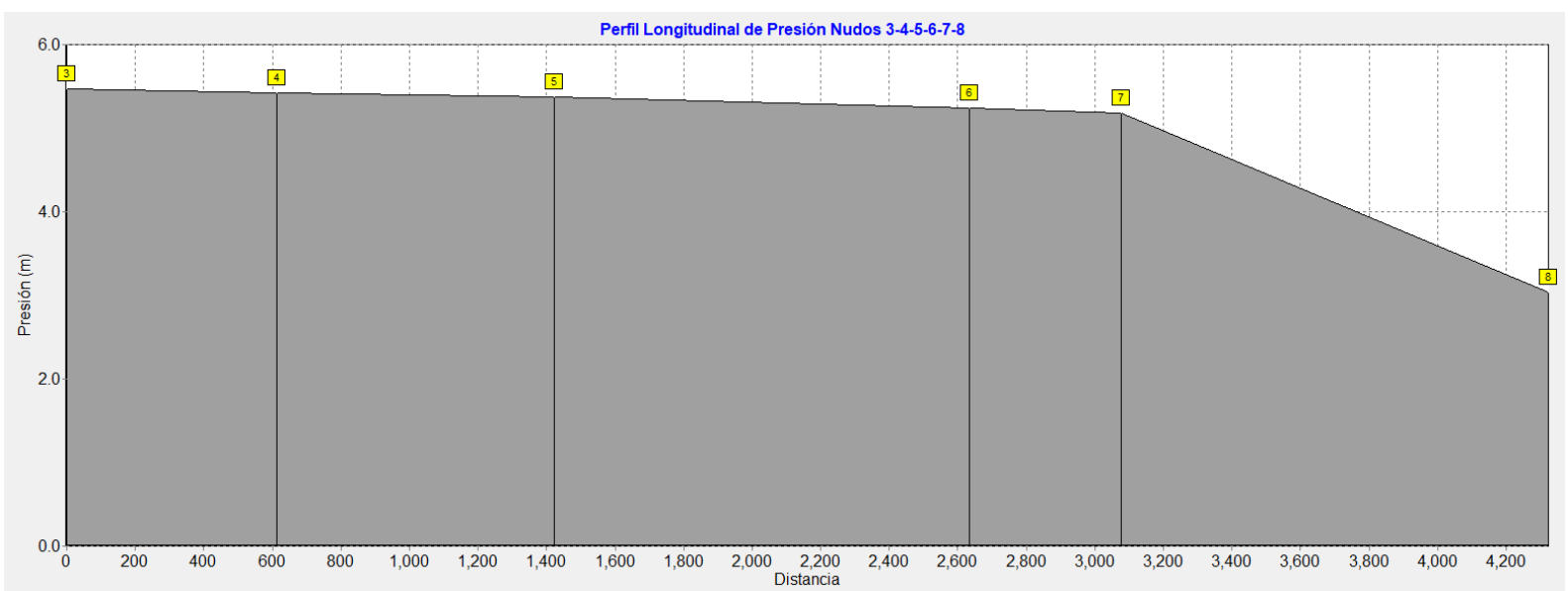

Figura 5 Presión en los nudos 3-4-5-6-7-8 
En la Figura 6, se observa que la presión en los nudos $11,12,13,14,15$ y 16, es mayor a la mínima recomendada.

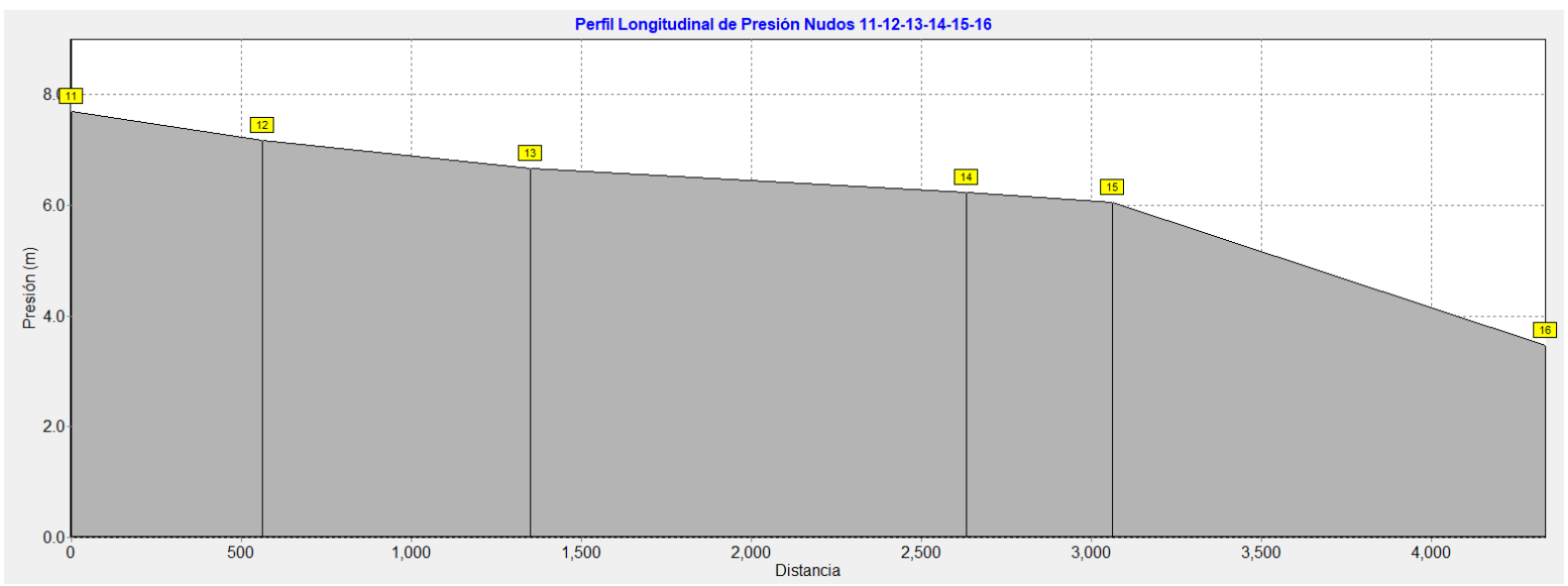

En la Figura 7 , se observa que la presión en los nudos $19,20,21,22,23$ y 24 , es mayor a la mínima recomendada.

Figura 8 Presión en los nudos 11-12-13-14-15-16

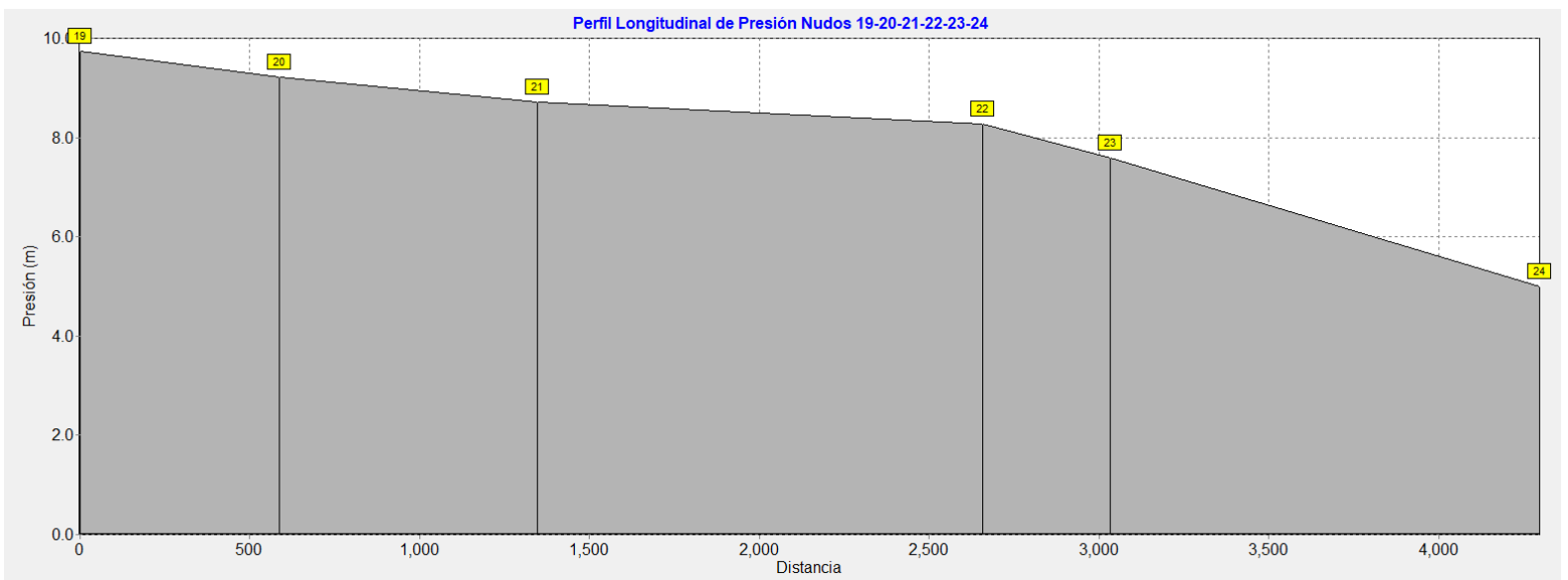

Figura 9 Presión en los nudos 19-20-21-22-23-24 


\section{Conclusiones}

Se comprobó que el software EPANET permite la simulación de redes de distribución de agua potable en edificios, introduciendo un artificio de cálculo con un caudal ficticio en los nudos, para cumplir con la ecuación de conservación de la masa en cada nudo.

El método fue probado en una red para un edificio de tres pisos, calculando las pérdidas de carga, velocidades y presiones, obteniendo los resultados con gran facilidad en un entorno grafico utilizando diferentes colores, lo que permite analizar cada uno de los resultados en varios escenarios posibles. El programa podría ser utilizado para modelar la red de distribución de agua de cualquier edificación sin ninguna limitación en el número de nudos.

En este estudio se elaboraron gráficos que muestran los valores de la presión en cada ramal de la red, que permitieron analizar el cumplimiento de las presiones mínimas contempladas en la normativa vigente.

Los resultados de la modelación en EPANET, pueden ser exportados como tablas, gráficos, curvas a otros programas, permitiendo al diseñador elaborar las memorias de cálculo de forma rápida. 


\section{Referencias Bibliográficas}

Adeniran, A., \& Oyelowo, M. (2014). An EPANET Analysis of Water Distribution Network of the University of Lagos, Nigeria. Journal of Engineering Research.

Aguirre, F. (2015). Abastecimiento de agua para comunidades rurales. Machala: Universidad Tecnica de Machala.

Blokker, E., Vreeburg, S., Buchberger, S., \& van Dijk, J. (2008). Importance of demand modelling in network water. Drinking water engineering and science.

Cabrera Bejar, J., \& Gueorguiev Tzatchkov, V. (2012). Modelación de redes de distribución de agua con suministro intermitente. Tecnología y Ciencias del Agua, 5-25.

Conety, S., Renganathan, N., Perumal, S., \& Paez, D. (2019). Analysis of water distribution network under pressure-deficient conditions through emitter setting. Drinking water engineering and science.

Mehta, D., Lakhani, K., Patel, D., \& Patel, G. (2015). Study of water distribution network using EPANET. Engineering: Issues, opportunities and Challenges for Development.

Miranda Trejo, M., Ocampo Fletes, I., Escobedo Castillo, J., \& Hernandez Rodriguez, M. (2015). La distribución del agua potable en Tepexi de Rodríguez, Puebla. Agricultura, Sociedad y Desarrollo, 261-277.

Morelos, R., \& Ramírez, J. (2017). Modelación hidráulica de la red de distribución de agua potable en una ciudad Mexicana EPANET . Revista Iberoamericana de Ciencias , 120-133.

NEC. (2011). Norma Hidrosanitaria NHE Agua. En C. e. Construccion, Norma Ecuatoriana de la Construccion (pág. 38). Quito.

Saldarriaga , J. (2014). Modelo estocastico de demanda de agua en edificaciones y la recoleccion y procesamieno de datos para su formulacion. Revista de ingenieria, Universidad de Los Andes, 33-38.

Saldarriaga, J. (2015). Hidráulica de Tuberías, Abastecimiento de Agua, Redes, Riegos. Bogota: Alfaomega.

Shivalingaswami, S., Vijaykumar, H., \& Nagaraj, P. (2016). Hydraulic modeling of water supply network using EPANET. International Research Journal of Engineering and Technology.

Venkata, G., Sudheer, C., \& Rajasekhar, B. (2015). Network analysis of water distribution system in rural areas using EPANET. Computer Control for Water Industry, 496-505.

Venkata, G., Sudheer, V., \& Pradad, L. (2016). Hydraulic Simulation of Existing Water Distribution System using EPANET. Indian Journal of Science and Technology. 